Le document est disponible dans la thèse en version article accepté à partir de la page 132 à l'adresse URL suivante : http://espace.inrs.ca/id/eprint/2746

Cellulose (2014) 21:3457-3468

DOI 10.1007/s10570-014-0361-9

ORIGINAL PAPER

\title{
Optimization of microfluidization for the homogeneous distribution of cellulose nanocrystals (CNCs) in biopolymeric matrix
}

\author{
Avik Khan · Khanh Dang Vu • Gregory Chauve • \\ Jean Bouchard • Bernard Riedl • \\ Monique Lacroix
}

Received: 8 May 2014/Accepted: 15 June 2014/Published online: 19 July 2014 (C) Springer Science+Business Media Dordrecht 2014

\begin{abstract}
Microfluidization, which is a high-pressure homogenization technique, was used to develop highly dispersed cellulose nanocrystal (CNC) reinforced chitosan based nanocomposite films. A three factor central composite design with five levels was designed to systematically optimize the microfluidization process. The three factors were the CNC content, the microfluidization pressure and the number of microfluidization cycles. Response surface methodology was used to obtain relationship between the mechanical properties of the nanocomposite films and the factors. Polynomial
\end{abstract}

Microscopic analysis of the microfluidized nanocomposite films revealed a $10-15$ times reduction in the size of the aggregates compared to the non-microfluidized $\mathrm{CNC} /$ chitosan films and an increase in the root mean square surface roughness $(\mathrm{Rq})$.

Keywords Chitosan - Cellulose nanocrystal Bionanocomposite - Experimental design · Highpressure-homogenization - Mechanical property . Nanomaterial dispersion - Polyelectrolyte complex 